\title{
The protective effect of SARS-CoV-2 antibodies in Scottish healthcare workers
}

\author{
Hani Abo-Leyah ${ }^{1}$ Stephanie Gallant ${ }^{1}$, Diane Cassidy ${ }^{1}$, Yan Hui Giam¹, \\ Justin Killick ${ }^{2}$, Beth Marshall², Gordon Hay ${ }^{2}$, Caroline Snowdon ${ }^{3}$, \\ Eleanor J. Hothersall ${ }^{3}$, Thomas Pembridge ${ }^{1}$, Rachel Strachan ${ }^{1}$, \\ Natalie Gallant ${ }^{1}$, Benjamin J. Parcell ${ }^{4}$, Jacob George ${ }^{1}$, Elizabeth Furrie ${ }^{2,5}$ and \\ James D. Chalmers ${ }^{1,5}$
}

Affiliations: ${ }^{1}$ Division of Molecular and Clinical Medicine, Ninewells Hospital and Medical School, Dundee, UK. ${ }^{2}$ Dept of Immunology, NHS Tayside, Ninewells Hospital and Medical School, Dundee, UK. ${ }^{3}$ Dept of Public Health, NHS Tayside, Ninewells Hospital and Medical School, Dundee, UK. ${ }^{4}$ Dept of Microbiology, NHS Tayside, Ninewells Hospital and Medical School, Dundee, UK. ${ }^{5}$ These authors contributed equally.

Correspondence: James D. Chalmers, University of Dundee, Ninewells Hospital and Medical School, DD1 9SY, UK. E-mail: j.chalmersadundee.ac.uk

\section{ABSTRACT}

Background: Healthcare workers (HCWs) are believed to be at increased risk of severe acute respiratory syndrome coronavirus 2 (SARS-CoV-2) infection. It is not known to what extent the natural production of antibodies to SARS-CoV-2 is protective against re-infection.

Methods: A prospective observational study of HCWs in Scotland (UK) from May to September 2020 was performed. The Siemens SARS-CoV-2 total antibody assay was used to establish seroprevalence in this cohort. Controls, matched for age and sex to the general local population, were studied for comparison. New infections (up to 2 December 2020) post antibody testing were recorded to determine whether the presence of SARS-CoV-2 antibodies protects against re-infection.

Results: A total of 2063 health and social care workers were recruited for this study. At enrolment, 300 HCWs had a positive antibody test (14.5\%). 11 out of 231 control sera tested positive (4.8\%). HCWs therefore had an increased likelihood of a positive test (OR 3.4, 95\% CI 1.85-6.16; p<0.0001). Dentists were most likely to test positive. $97.3 \%$ of patients who had previously tested positive for SARS-CoV-2 by reverse transcriptase (RT)-PCR had positive antibodies. $18.7 \%$ had an asymptomatic infection. There were 38 new infections with SARS-CoV-2 in HCWs who were previously antibody negative, and one symptomatic RT-PCR-positive re-infection. The presence of antibodies was therefore associated with an $85 \%$ reduced risk of re-infection with SARS-CoV-2 (hazard ratio 0.15, 95\% CI 0.06-0.35; $\mathrm{p}=0.026$ ).

Conclusion: HCWs were three times more likely to test positive for SARS-CoV-2 than the general population. Almost all infected individuals developed an antibody response, which was $85 \%$ effective in protecting against re-infection with SARS-CoV-2.

@ERSpublications

In this study, healthcare workers were three times more likely to test positive for \#SARSCoV2 than the general population. Almost all infected individuals developed an antibody response, and this was $85 \%$ effective in protecting against re-infection. https://bit.ly/3mLPUmk

Cite this article as: Abo-Leyah $\mathrm{H}$, Gallant $\mathrm{S}$, Cassidy D, et al. The protective effect of SARS-CoV-2 antibodies in Scottish healthcare workers. ERJ Open Res 2021; 7: 00080-2021 [https://doi.org/ 10.1183/23120541.00080-2021]. 


\section{Introduction}

Healthcare workers (HCWs) are known to be at increased risk of symptomatic infection with severe acute respiratory syndrome coronavirus 2 (SARS-CoV-2) [1,2]. HCWs accounted for $21 \%$ of SARS cases during the outbreak in 2002 [3] and high rates of symptomatic infections have been reported across Europe during the present pandemic, including in the UK [4]. Measures taken to mitigate this increased risk include adequate personal protective equipment (PPE) [5], infection prevention and control (IPC) procedures within healthcare environments and staff testing. Across the UK, for healthcare and other key workers with symptoms, testing has been widely available since April 2020 [6].

A key challenge in containing the spread of SARS-CoV-2 has been the potential for asymptomatic or atypical infection [7]. Even in the case of symptomatic individuals, reverse transcriptase (RT)-PCR on nasopharyngeal, oropharyngeal or combined upper airway swabs has a reported sensitivity of 70-90\% and consequently will underestimate the number of infected individuals [8]. Therefore, the extent of infections in HCWs in different parts of the world remain largely unknown.

Serological testing can be used to determine the incidence and prevalence of SARS-CoV-2 infection [9]. Identifying the extent of HCW infections and the proportion of undetected infections is important to inform IPC measures during future waves of the pandemic.

An antibody response is expected after infection with SARS-CoV-2 but the rate of antibody development has not been extensively reported. Little is known about the protective effect of natural immunity and no studies have been published that demonstrate how protective natural antibodies are against re-infection with SARS-CoV-2.

In this study, we investigated the seroprevalence of SARS-CoV-2 antibodies in a large population of Scottish HCWs. We also investigated whether the presence of antibodies protects against re-infection with the virus.

\section{Methods}

We conducted a prospective observational study recruiting HCWs employed within the National Health Service in Tayside (NHS Tayside). NHS Tayside is an NHS board in the East of Scotland (UK) that is responsible for delivering healthcare for over 400000 people and employs around 14000 staff.

Healthcare staff were invited to participate in the study via advertisements, including e-mail newsletters and posted adverts on the staff intranet page. Recruitment took place during a single study visit at Ninewells Hospital (Dundee, UK), which is the health board's largest teaching hospital. Recruitment took place between 28 May 2020 and 2 September 2020. Electronic results were followed up until 2 December 2020, to record new laboratory-confirmed infections. All participants gave written informed consent to participate. The study was approved by the West of Scotland Research Ethics committee, approval number 20/WS/0078.

The inclusion criteria were: employment as a health or social care worker, and age $>16$ years. Participants were excluded if they had any contraindication to venepuncture, symptoms consistent with current SARS-CoV-2 infection at the time of enrolment, or had tested positive for SARS-CoV-2 in the preceding 14 days.

At the study visit, participants completed a questionnaire on demographics, previous symptoms, employment role, hours of work, contact with patients with coronavirus disease 2019 (COVID-19) infection and whether they had previously tested positive for SARS-CoV-2. Blood samples were taken for measurement of SARS-CoV-2 antibodies in serum.

\section{SARS-CoV-2 antibody detection}

The Siemens SARS-CoV-2 total antibody assay was used in this study. This is a one stop bridging chemiluminescent immunoassay method that detects antibodies against the receptor-binding domain of the SARS-CoV-2 spike (S1) protein. The assay is performed on the Siemens Atellica 1300 platform. Validation of this assay was approved by the NHS Scotland national laboratories programme quality group and was then further validated against other commercial antibody platforms in a previous study and found to have $95-100 \%$ sensitivity, while titres remained constant beyond 81 days following a positive PCR test result [10].

\section{Population control subjects}

A random selection of blood samples taken at NHS Tayside General Practice Surgeries were tested, covering the same time period as the HCW study cohort. Samples were age and sex matched to the 
Scottish population demographics to provide a representative sample of the local population, to determine the background prevalence of SARS-CoV-2. Serum samples were run on the same Siemens analyser.

\section{Follow-up}

All individuals in Scotland have a unique identifier number (community health index) that links to their healthcare records. Using these identifiers, participants' antibody data were linked to results of symptomatic testing for SARS-CoV-2 at regional and national laboratories. Immediate testing for HCWs displaying symptoms is available in the study region, ensuring all symptomatic SARS-CoV-2 infections would be captured during follow-up until 2 December 2020.

\section{Statistical analysis}

Data were analysed using IBM SPSS v25 and GraphPad Prism 8.1.2. Chi-squared and Fisher's tests were used as appropriate to compare proportions between groups. Logistic regression was used to derive the odds ratio values for the reported symptom analysis. Kaplan-Meier survival analysis with follow-up from date of antibody testing until 2 December 2020 was used with groups compared using the log-rank (Mantel-Cox) test. A p-value $<0.05$ was considered statistically significant for all analyses.

\section{Results}

A total of 2063 health and social care workers were recruited for this study. The participants were predominantly female $(81.7 \%)$ and $95.5 \%$ were white. The median age of participants was 46 years. Table 1 presents the demographic characteristics of the study participants and their healthcare roles.

\section{Seroprevalence of SARS-CoV-2 antibodies}

In our study, $300 \mathrm{HCW}$ had a positive antibody test directed against SARS-CoV-2 spike protein. This represents a seroprevalence of 14.5\%. 11 out of 231 control sera tested positive (4.8\%), which was consistent with the broader Scottish surveillance data reported by Health Protection Scotland (214 positive tests out of $4751 ; 4.5 \%$ ). Compared to both sets of population controls, HCWs had 3.6 times greater odds of a positive test compared to local controls (OR 3.4, 95\% CI 1.85-6.16; p<0.0001) and compared to Scotland-wide controls (OR 3.6, 95\% CI 2.99-4.32; p<0.001).

Table 1 shows the seroprevalence rate among subgroups in our study characterised by demographic, job role and area of work. Male sex was more frequently associated with detected antibodies (18.5\% versus $13.7 \% ; \mathrm{p}=0.02$ ). Some job roles were significantly associated with a higher rate of SARS-CoV-2 antibody detection. HCWs in dentistry were the most frequently associated with detected antibodies (26\%), followed by healthcare assistants $(23.3 \%)$ and hospital porters $(22.2 \%)$ ( $\mathrm{p}<0.0001$ when comparing across groups). Figure 1 displays the rates of antibody prevalence among the HCWs, by profession.

Healthcare staff who worked in areas of the hospital that treated suspected or confirmed cases of COVID-19 were more frequently associated with detected antibodies (17.4\% versus $13.5 \%$; $\mathrm{p}=0.03)$. Staff who worked in critical care and the intensive care unit were not more frequently associated with detected antibodies ( $16 \%$ versus $14.4 \%$; $\mathrm{p}=0.61)$.

\section{Prior positive test results and symptomatic infections}

797 study participants had a SARS-CoV-2 RT-PCR swab prior to enrolment into the study and therefore prior to antibody measurement. 111 of these were positive for SARS-CoV-2 prior to enrolment. 97.3\% (108 out of 111) of participants with positive RT-PCR had detectable antibodies. $10.9 \%$ of participants with a negative PCR test prior to enrolment had detectable antibody levels. In those who never had an RT-PCR test, $8.9 \%$ had detectable antibodies. Figure 2 displays the proportions of PCR-positive and PCR-negative participants with detected antibodies.

45.4\% ( $\mathrm{n}=936$ ) of the HCWs recruited believed they had had COVID-19 but only $25.1 \%$ ( $\mathrm{n}=235$ ) of these HCWs had detectable antibodies. Conversely, $18.7 \%(n=56)$ of those who had antibodies detected did not believe they ever had had COVID-19 and were completely asymptomatic.

$5.1 \%(n=56)$ of asymptomatic participants had detectable antibodies compared to $25.6 \%(n=243)$ of symptomatic recruits who had detectable antibodies. When compared with the general population, individuals who did not have a symptomatic illness during the period of the study did not have an increased frequency of SARS-CoV-2 antibodies (OR 1.20, 95\% CI 0.30-4.83; p=0.26).

Anosmia was the self-reported symptom that was most likely to correspond with detected antibodies (OR 12.3, 95\% CI 9.3-16.3; p<0.001) but was only reported in 5.8\% of HCWs at any time (table 2). A combination of cough, fever and anosmia was only reported at a frequency of $2.6 \%$ but when present was associated with a 10-fold increase in odds of detectable antibody (OR 9.7, 95\% CI 6.4-14.7; p<0.001). 


\begin{tabular}{|c|c|c|c|c|}
\hline Characteristic & $\mathrm{n}(\%$ of total $)$ & Antibody positive $\mathrm{n}$ & Seroprevalence \% & p-value \\
\hline \multicolumn{4}{|l|}{ Sex } & $0.02 *$ \\
\hline Male & 378 (18.3) & 70 & 18.5 & \\
\hline Female & $1685(81.7)$ & 230 & 13.7 & \\
\hline \multicolumn{4}{|l|}{ Age years } & 0.5 \\
\hline $18-30$ & $290(14.1)$ & 46 & 15.9 & \\
\hline $31-40$ & 403 (19.5) & 60 & 14.9 & \\
\hline $41-50$ & $536(26.0)$ & 76 & 14.2 & \\
\hline $51-60$ & 637 (30.9) & 97 & 15.2 & \\
\hline$>60$ & $196(9.5)$ & 21 & 10.7 & \\
\hline \multicolumn{4}{|l|}{ Ethnicity } & 0.86 \\
\hline White European & 1964 (95.5) & 285 & 14.5 & \\
\hline Other White & $16(0.8)$ & 2 & 12.5 & \\
\hline South Asian & $36(1.7)$ & 8 & 22.2 & \\
\hline Chinese & $10(0.5)$ & 1 & 10.0 & \\
\hline Black & $8(0.4)$ & 2 & 25.0 & \\
\hline Arab & $5(0.2)$ & 1 & 20.0 & \\
\hline Traveller & $2(0.1)$ & 0 & 0.0 & \\
\hline Other ethnic & $16(0.8)$ & 1 & 6.3 & \\
\hline \multicolumn{4}{|l|}{ Role } & $<0.0001^{*}$ \\
\hline Doctor & $237(11.5)$ & 50 & 21.1 & \\
\hline Nurse & $601(29.2)$ & 80 & 13.3 & \\
\hline $\mathrm{AHP}$ & $239(11.6)$ & 25 & 10.5 & \\
\hline Pharmacy staff & $69(3.4)$ & 9 & 13.0 & \\
\hline $\mathrm{HCA}$ & $172(8.4)$ & 40 & 23.3 & \\
\hline Student & $25(1.2)$ & 4 & 16.0 & \\
\hline Domestic & $84(4.1)$ & 11 & 13.1 & \\
\hline Admin & $403(19.6)$ & 49 & 21.1 & \\
\hline Porter & $27(1.3)$ & 6 & 22.2 & \\
\hline Other ${ }^{\#}$ & 151 (7.3) & 13 & 8.6 & \\
\hline Dentistry & $50(2.4)$ & 13 & 26.0 & \\
\hline \multicolumn{4}{|l|}{ Area } & $0.03^{*}$ \\
\hline COVID-19 & $552(26.8)$ & 96 & 17.4 & \\
\hline Non-COVID-19 & $1511(73.2)$ & 204 & 13.5 & \\
\hline \multicolumn{4}{|l|}{ Critical care } & 0.61 \\
\hline Yes & $131(6.3)$ & 21 & 16.0 & \\
\hline No & 1931 (93.7) & 279 & 14.4 & \\
\hline \multicolumn{5}{|c|}{$\begin{array}{l}\text { AHP: allied health professional; HCA: healthcare assistant; Admin: administrative staff. \#: other roles } \\
\text { included lab technician, health scientist, maintenance, laundry, medical physics, other technician, patient } \\
\text { transport, chaplaincy, volunteers. Chi-squared and Fisher's exact tests were used to determine statistical } \\
\text { significance. }{ }^{*}: \text { p }<0.05 \text {, statistically significant. }\end{array}$} \\
\hline
\end{tabular}

The absence of any cough, fever and anosmia was associated with an $82 \%$ chance of not having detectable antibodies (OR 0.2, 95\% CI 0.14-0.24; $\mathrm{p}<0.001$ ).

\section{Infection rate and antibody protection during second wave of infections}

In the 3 months following the end of the recruitment period, there were 39 new symptomatic infections with SARS-CoV-2. 38 of these 39 infections were in HCWs who did not have prior antibodies to SARS-CoV-2.

There was only one re-infection ( 1 out of $300 ; 0.33 \%$ ) with SARS-CoV-2. This re-infection was in a symptomatic HCW who tested positive by RT-PCR 76 days after having detectable antibodies in their serum.

From the first recorded PCR-positive infection in this cohort to the end of the follow-up period was 261 days and from the first positive antibody test to the end of the follow-up period was 188 days. Figure 3 shows an analysis of time from antibody testing to development of PCR-confirmed symptomatic SARS-CoV-2 infection. This demonstrates that having antibodies to SARS-CoV-2 at baseline is highly protective against re-infection (hazard ratio $0.15,95 \%$ CI $0.06-0.35 ; \mathrm{p}=0.026$ ) over a follow-up period of up to 6 months. 
FIGURE 1 Percentage prevalence of SARS-CoV-2 antibody among different healthcare worker roles. Staff working in dentistry had the highest prevalence. AHP: allied health professional; Admin: administrative staff; HCA: healthcare assistant.

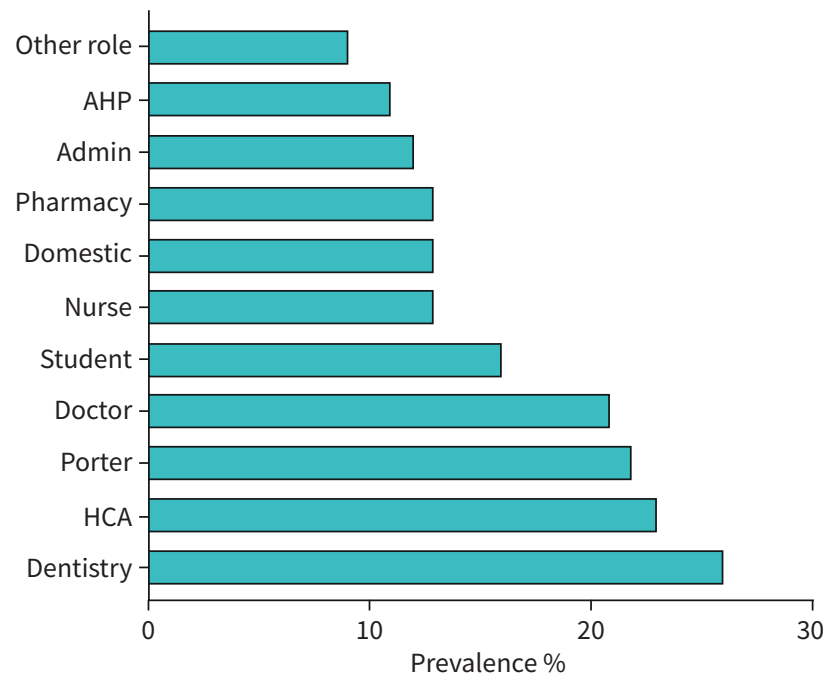

\section{Discussion}

In this observational study of health and social care workers, the SARS-CoV-2 seroprevalence rate was $14.5 \%$. Importantly, the presence of antibodies was associated with clear protection against subsequent infection. This is important for our future understanding of risk for HCWs and the general population.

At the time of writing, Health Protection Scotland is leading a surveillance study for COVID-19 in Scotland on behalf of the Scottish Government [11]. Present data from this study demonstrate a comparative seroprevalence of $4.5 \%$. Our data therefore suggest that health and social care workers are $>3$ times more likely to be positive for spike protein antibodies and thus are likely to have been infected with SARS-CoV-2.

Other HCW seroprevalence studies conducted in the rest of the UK have also reported higher seropositivity when compared to the general population, with seroprevalence rates of $24.4 \%$ [12], $25.4 \%$ [13] and $10.7 \%$ [14].

Our study was notable for the inclusion of dentistry staff, who had the highest seroprevalence rate at $26 \%$. This was well above the average seroprevalence rate of $14.5 \%$ among our HCWs, although this higher rate should be interpreted with some caution given the lower number of dentistry staff enrolled (table 1). Dentistry staff are expected to be a higher risk group, given their focus of work is more likely to be aerosol generating and with close exposure to potentially infected mucosal surfaces [15]. Healthcare assistants, including such staff at nursing homes, had the second highest prevalence at $23 \%$. The caring roles of these workers necessitate close patient contact and their increased risk was also reported in a recent Swedish study [16].

In other published HCW studies [12], domestic or housekeeping staff had the highest seroprevalence of antibodies. This was not evident in our study, where domestic staff had a below average seroprevalence rate of $13.1 \%$. Other groups who had a seroprevalence rate above average were hospital porters and doctors. Doctors and porters are typically exposed to multiple patients in different working areas.

FIGURE 2 Proportion of antibody detection according to PCR status. $97.3 \%$ of PCR-positive individuals had detectable antibodies. $10.9 \%$ of PCR-negative participants had detectable antibodies.
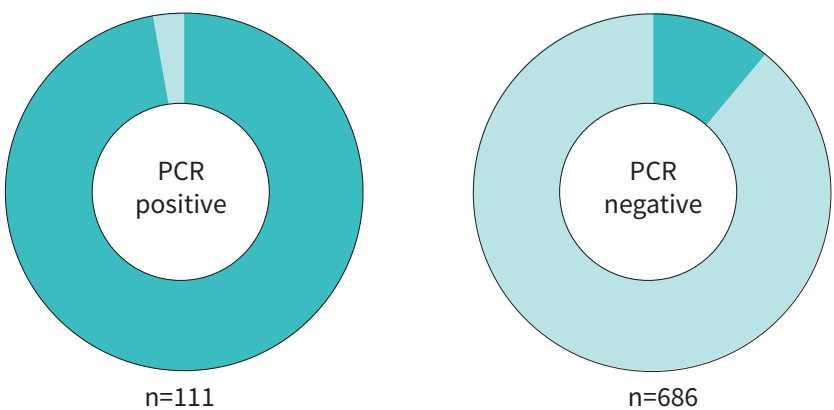

Antibody positive

Antibody negative 
TABLE 2 Frequency of reported symptoms and odds ratios of each symptom corresponding to detectable antibodies against SARS-CoV-2

\begin{tabular}{lccc} 
Symptoms & Frequency $\%$ & OR (95\% CI) & p-value \\
\hline Fatigue & 15.4 & $4.5(3.458-5.912)$ & 0.0001 \\
Headache & 12.3 & $3.6(2.809-4.653)$ & 0.0001 \\
Cough & 11.8 & $2.1(1.619-2.67)$ & 0.0001 \\
Myalgia & 11.7 & $3.8(2.931-4.856)$ & 0.0001 \\
Fever & 10.0 & $2.5(1.74-2.901)$ & 0.0001 \\
Sore throat & 9.8 & $1.4(1.092-1.86)$ & 0.009 \\
Dyspnoea & 9.2 & $2.4(1.825-3.076)$ & 0.0001 \\
Runny nose & 5.9 & $1.2(0.835-1.61)$ & 0.378 \\
Anosmia & 5.8 & $12.3(9.306-16.348)$ & 0.0001 \\
Other & 5.0 & $3.5(2.615-4.705)$ & 0.001 \\
Diarrhoea & 3.1 & $2.5(1.773-3.66)$ & 0.0001 \\
CFA & 2.6 & $9.7(6.433-14.738)$ & 0.0001 \\
Absence of CFA & $0.2(0.136-0.239)$ & 0.001 \\
\hline CFA: combination of cough, fever and anosmia. & & \\
\hline
\end{tabular}

By comparison, nurses typically care for up to six patients in a defined area during their working day. The variability in work location in a particular time period could be a factor in the increased infection rate we observed.

In our study, HCWs who worked in COVID-19 areas of the hospital had a slightly higher seroprevalence than those workers who did not. This finding is consistent with other studies, including HCW studies conducted in major urban areas where the community burden of COVID-19 was a significant source of exposure [17]. Working in COVID-19 areas of the hospital is one way to define high-risk exposure. Nevertheless, the majority of SARS-CoV-2 infections detected in this study occurred in staff who were not working directly with COVID-19 patients, and this group still had a significantly higher prevalence of SARS-CoV-2 antibodies than the general population. While a seroprevalence study cannot establish the source of infection, this strongly suggests transmission between healthcare staff within non-clinical environments, since many staff roles that did not involve direct contact with patients were still associated with an increased rate of antibody positivity. Therefore, while much media attention has focused on the importance of PPE for front-line staff, these data emphasise the importance of IPC measures in non-clinical areas within healthcare environments such as hospitals. The relative success of measures to protect high-risk front-line staff is illustrated by the low rate of antibody detection in critical care staff. All staff working in critical care areas wore PPE in accordance with Health Protection Scotland guidance on working in aerosol-generating procedures [18]. We found no significantly increased risk of infection for these staff. In the recently published study from Birmingham, UK [12], staff in intensive care had a significantly lower risk of seropositivity.

We asked our study participants to report if they thought they had had COVID-19 and list the symptoms they experienced. We describe that only one in four participants who thought they had contracted

FIGURE 3 Survival curve showing protective effect of pre-existing antibodies to developing new SARS-CoV-2 infections. Those who had previously detected antibodies to SARS-CoV-2 were protected against re-infection.

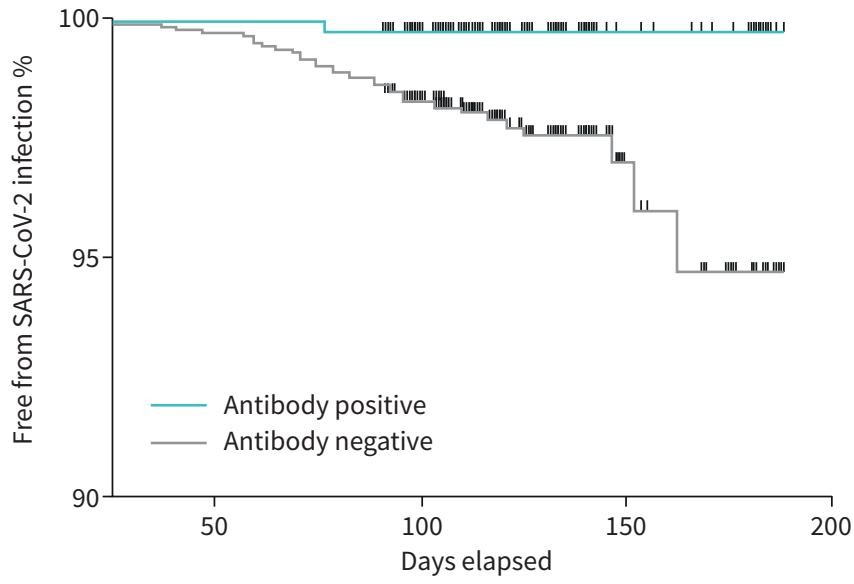


COVID-19 demonstrated serological evidence of infection. The heightened suspicion of infection is justified among HCWs, but perceived infection does not correlate well with actual infection. This has some potentially important implications when considering the issue of chronic symptoms in individuals who believe they have had COVID-19 infection [19]. However, we demonstrated in this study that certain symptoms are significantly more predictive of infection with SARS-CoV-2. Anosmia represented 12-fold increased odds of having serological evidence of infection. This particular symptom was also shown to be strongly predictive in similar analysis performed in other European studies [16, 20].

Our study demonstrates that approximately one-fifth (18.7\%) of seropositive HCWs were completely asymptomatic. This is consistent with studies that only recruited asymptomatic HCWs [12] and indicates that a significant proportion of the healthcare workforce will attend work without knowing that they may potentially transmit the infection to their colleagues.

After the end of the recruitment period, we followed our study participants for up to 6 months from antibody positivity and up to 10 months from first PCR-confirmed SARS-CoV-2 infection. Winter 2020 coincided with the second wave of SARS-CoV-2 infections in Scotland and we had 39 new infections among our HCWs during this period. One of these infections ( $0.33 \%$ of the total study group) was a symptomatic re-infection in an HCW who had previously developed an antibody response from a previous infection. To our knowledge, this is the first reported case of laboratory-confirmed re-infection after the development and confirmed presence of natural antibodies.

97.4\% of new infections were in HCWs who did not have previous antibodies. In our study, the presence of a natural antibody response was therefore $85 \%$ protective against re-infection with SARS-CoV-2 over a period of 6 months (May to December 2020).

Our results on antibody protection fit within the range of efficacy of vaccine-mediated immunity reported in the recently published SARS-CoV-2 vaccine trials [21, 22]. These results may have implications for the roll-out of vaccine programmes, since HCWs are often prioritised, but our results suggest HCWs with prior infection may be at low risk of future infection and may therefore be considered a lower priority.

This study has potential limitations, including potentially that individuals more likely to believe they have had a SARS-CoV-2-like illness would be more likely to volunteer for such a study. Nevertheless, we were successful in enrolling participants who had never experienced a symptomatic infection and demonstrate an increased seroprevalence even among this group. We enrolled patients up to September 2020 and therefore potentially up to $4-5$ months post infection. This raises the possibility of antibodies waning over time [23]. This seems unlikely, as a prior study found no evidence of waning of the Siemens assay over 4 months [10], while a similar total spike protein antibody assay showed no waning over time in a study from Iceland [24]. Other studies have reported increased infection rates in the Black, Asian and minority ethnic populations [25-27]. We were unable to investigate this as NHS Tayside has a workforce that is $97 \%$ white. The patient population was representative of NHS Scotland staff. In 2019, the median age of staff employed across NHS Scotland was 46 years, and $77.4 \%$ of NHS Scotland employees were female. $9 \%$ were doctors, $0.4 \%$ dental staff, $42.6 \%$ nursing staff, $8.4 \%$ allied health professionals and $18.1 \%$ administrative staff. Our sample included $11.5 \%$ doctors, $29.2 \%$ nurses, $11.6 \%$ allied health professionals and $19.6 \%$ administrative staff. Our median age was 46 years and $81.7 \%$ of our cohort were female.

\section{Conclusion}

Our study suggests that HCWs are at increased risk of infection with SARS-CoV-2 compared with the general population. Our study suggests a differential risk among hospital staff and a high proportion of undetected symptomatic and asymptomatic infections. This will help to inform targeted IPC strategies during future epidemics. We report a single re-infection in an HCW who had developed natural antibodies and we estimate the natural antibody response is $85 \%$ protective against re-infection.

Author contributions: S. Gallant, D. Cassidy, B.J. Parcell, J. George, E. Furrie and J.D. Chalmers designed and conducted the study; H. Abo-Leyah, S. Gallant, D. Cassidy, R. Strachan, N. Gallant, C. Snowdon, E.J. Hothersall, J. Killick, E. Furrie and J.D. Chalmers collected data; D. Cassidy, Y.H. Giam, J. Killick, B. Marshall, G. Hay, T. Pembridge, R. Strachan and E. Furrie performed laboratory work; H. Abo-Leyah and J.D. Chalmers wrote the manuscript; and all authors reviewed the manuscript and approved the final version.

Availability of data and materials: Data and materials can be made available upon reasonable request and in keeping with our ethics and data protection protocols.

Conflict of interest: H. Abo-Leyah has nothing to disclose. S. Gallant has nothing to disclose. D. Cassidy has nothing to disclose. Y.H. Giam has nothing to disclose. J. Killick has nothing to disclose. B. Marshall has nothing to disclose G. Hay has nothing to disclose. C. Snowdon has nothing to disclose. E.J. Hothersall has nothing to disclose. T. Pembridge has nothing to disclose. R. Strachan has nothing to disclose. N. Gallant has nothing to disclose 
B.J. Parcell has nothing to disclose. J. George has nothing to disclose. E. Furrie has nothing to disclose. J.D. Chalmers reports grants and personal fees from AstraZeneca, Boehringer Ingelheim, GlaxoSmithKline and Insmed; personal fees from Chiesi, Novartis and Zambon; and grants from Gilead Sciences, all outside the submitted work.

Support statement: This study was supported by the NHS Tayside COVID-19 Research Fund. J.D. Chalmers is supported by the British Lung Foundation Chair of Respiratory Research. Funding information for this article has been deposited with the Crossref Funder Registry.

\section{References}

1 Alhazzani W, Møller MH, Arabi YM, et al. Surviving Sepsis Campaign: guidelines on the management of critically ill adults with coronavirus disease 2019 (COVID-19). Crit Care Med 2020; 48: e440-e469.

2 Livingston E, Bucher K. Coronavirus disease 2019 (COVID-19) in Italy. JAMA 2020; 323: 1335.

3 Xiao J, Fang M, Chen Q, et al. SARS, MERS and COVID-19 among healthcare workers: a narrative review. J Infect Public Health 2020; 13: 843-848.

4 Nguyen LH, Drew DA, Graham MS, et al. Risk of COVID-19 among front-line health-care workers and the general community: a prospective cohort study. Lancet Public Health 2020; 5: e475-e483.

5 Thomas JP, Srinivasan A, Wickramarachchi CS, et al. Evaluating the national PPE guidance for NHS healthcare workers during the COVID-19 pandemic. Clin Med 2020; 20: 242-247.

6 Parcell BJ, Brechin K, Allstaff S, et al. Drive-through testing for SARS-CoV-2 in symptomatic health and social care workers and household members: an observational cohort study. Thorax 2020; 75: 1109-1111.

7 Gandhi M, Yokoe DS, Havlir DV. Asymptomatic transmission, the Achilles' heel of current strategies to control COVID-19. N Engl J Med 2020; 382: 2158-2160.

8 Woloshin S, Patel N, Kesselheim AS. False negative tests for SARS-CoV-2 infection - challenges and implications. N Engl J Med 2020; 383: e38.

9 Peeling RW, Wedderburn CJ, Garcia PJ, et al. Serology testing in the COVID-19 pandemic response. Lancet Infect Dis 2020; 20: e245-e249.

10 Muecksch F, Wise $\mathrm{H}$, Batchelor B, et al. Longitudinal analysis of clinical serology assay performance and neutralising antibody levels in COVID19 convalescents. medRxiv 2020; preprint [https://doi.org/10.1101/2020.08. 05.20169128].

11 Health Protection Scotland. Components of the programme (Enhanced Surveillance of COVID-19 in Scotland). www.hps.scot.nhs.uk/a-to-z-of-topics/enhanced-surveillance-of-coronavirus-covid-19/components-of-theprogramme/\#title-container Date last updated: 2020. Date last accessed: 29 September 2020.

12 Shields A, Faustini SE, Perez-Toledo M, et al. SARS-CoV-2 seroprevalence and asymptomatic viral carriage in healthcare workers: a cross-sectional study. Thorax 2020; 75: 1089-1094.

13 Houlihan CF, Vora N, Byrne T, et al. SARS-CoV-2 virus and antibodies in front-line health care workers in an acute hospital in London: preliminary results from a longitudinal study. medRxiv 2020; preprint [https://doi.org/ 10.1101/2020.06.08.20120584].

14 Eyre DW, Lumley SF, O’Donnell D, et al. Differential occupational risks to healthcare workers from SARS-CoV-2: a prospective observational study. medRxiv 2020; preprint [https://doi.org/10.1101/2020.06.24.20135038].

15 Coulthard P. Dentistry and coronavirus (COVID-19) - moral decision-making. Br Dent J 2020; 228: 503-505.

16 Rudberg A-S, Havervall S, Månberg A, et al. SARS-CoV-2 exposure, symptoms and seroprevalence in health care workers. medRxiv 2020; preprint [https://doi.org/10.1101/2020.06.22.20137646].

17 Wilkins JT, Gray EL, Wallia A, et al. Seroprevalence and correlates of SARS-CoV-2 antibodies in healthcare workers in Chicago. medRxiv 2020; preprint [https://doi.org/10.1101/2020.09.11.20192385].

18 Health Protection Scotland. PPE - For Unit Wide Airborne Precautions. https://hpspubsrepo.blob.core.windows. net/hps-website/nss/3006/documents/7_covid-19-ppe-unit-wide-airborne-precautions-a3.pdf Date last updated: November 2020. Date last accessed: 17 May 2021.

19 Rubin R. As their numbers grow, COVID-19 "long haulers" stump experts. JAMA 2020; 324: 1381-1383.

20 Garcia-Basteiro AL, Moncunill G, Tortajada M, et al. Seroprevalence of antibodies against SARS-CoV-2 among health care workers in a large Spanish reference hospital. Nat Commun 2020; 11: 3500.

21 Ramasamy MN, Minassian AM, Ewer KJ, et al. Safety and immunogenicity of ChAdOx1 nCoV-19 vaccine administered in a prime-boost regimen in young and old adults (COV002): a single-blind, randomised, controlled, phase 2/3 trial. Lancet 2020; 396: 1979-1993.

22 Polack FP, Thomas SJ, Kitchin N, et al. Safety and efficacy of the BNT162b2 mRNA COVID-19 vaccine. $N$ Engl J Med 2020; 383: 2603-2615.

23 Seow J, Graham C, Merrick B, et al. Longitudinal observation and decline of neutralizing antibody responses in the three months following SARS-CoV-2 infection in humans. Nat Microbiol 2020; 5: 1598-1607.

24 Gudbjartsson DF, Norddahl GL, Melsted P, et al. Humoral immune response to SARS-CoV-2 in Iceland. $N$ Engl J Med 2020; 383: 1724-1734.

25 Leeds JS, Raviprakash V, Jacques T, et al. Risk factors for detection of SARS-CoV-2 in healthcare workers during April 2020 in a UK hospital testing programme. EClinicalMedicine 2020; 26: 100513.

26 Ahmed MH. Black and minority ethnic (BAME) alliance against COVID-19: one step forward. $J$ Racial Ethn Health Disparities 2020; 7: 822-828.

27 Raisi-Estabragh Z, McCracken C, Bethell MS, et al. Greater risk of severe COVID-19 in Black, Asian and minority ethnic populations is not explained by cardiometabolic, socioeconomic or behavioural factors, or by 25 (OH)-vitamin D status: study of 1326 cases from the UK Biobank. J Public Health 2020; 42: 451-460. 